\title{
Obesity and Colorectal Cancer
}

\author{
Irena Gribovskaja-Rupp, M.D., ${ }^{1}$ Lauren Kosinski, M.D., ${ }^{1}$ and Kirk A. Ludwig, M.D. ${ }^{1}$
}

\begin{abstract}
Obesity is a risk factor for colorectal cancer based on its molecular and metabolic effects on insulin and IGF-1, leptin, adipocytokines, and sex hormones. Obese men have a higher risk of colorectal cancer compared with normal weight men, but the association between obesity and rectal cancer is weaker than with colon cancer. There is a weaker association between obesity and colon cancer in women than in men, and no appreciable association between obesity and rectal cancer in women. Although obesity does not seem to have an effect on the number of lymph nodes harvested with resection, obesity does seem to be associated with more-aggressive colorectal cancers in a handful of studies. Survival and local recurrence studies are contradictory with no conclusive evidence that obesity predisposes to worse overall survival or increased recurrence in colon and rectal cancers. The literature is not definitive as far as overall morbidity and mortality rates in the obese are concerned, though obese rectal cancer patients seem to incur proportionally more morbidity and mortality. Preexisting steatosis or steatohepatitis in obese colorectal cancer patients or chemotherapy-induced liver dysfunction may lead to an increased mortality in obese patients with colorectal liver metastases. Diabetes may cause poorer response to neoadjuvant therapy in rectal cancer and contribute to higher mortality and recurrence in colon cancer.
\end{abstract}

KEYWORDS: Obesity, colon cancer, rectal cancer, diabetes, men, women

Objectives: After reading this review, the reader should be able to summarize how and why obesity is a risk factor for colorectal cancer, and have an appreciation for how definitions of obesity vary and impact study results. In addition, the reader should also understand the impact of obesity on colorectal cancer short-term outcomes, long-term oncologic outcomes, and how obesity can impact the chemotherapeutic treatment of metastatic disease.

Colorectal cancer (CRC) is a major cause of cancer death in Western societies and it is well known that this elevated risk is related to an imbalance between neoplasia promoting and protective dietary and lifestyle factors. Among the factors that tend to elevate risk are a high intake of animal fat, high calorie intake, high intake of processed foods with low fiber content, limited physical activity, excess alcohol intake, and finally obesity.
What follows is a discussion on how and why obesity is a risk factor for colorectal cancer and how obesity influences short- and long-term oncologic outcomes from a surgical, pathologic, radiotherapy, and chemotherapeutic perspective. The data leads one to conclude that obesity has implications in all aspects of the care of colorectal cancer patients, from screening to surgical treatment to adjuvant or neoadjuvant treatments.

\footnotetext{
${ }^{1}$ Department of Surgery, Division of Colorectal Surgery, Medical College of Wisconsin, Milwaukee, Wisconsin.

Address for correspondence and reprint requests: Kirk A. Ludwig, M.D., Department of Surgery, Division of Colorectal Surgery, Medical College of Wisconsin, 9200 W. Wisconsin Ave., Milwaukee, WI 53226 (e-mail: kludwig@mcw.edu).

Colon and Rectal Surgery in the Obese Patient; Guest Editor,
}

\section{H. David Vargas, M.D.}

Clin Colon Rectal Surg 2011;24:229-243. Copyright (C) 2011 by Thieme Medical Publishers, Inc., 333 Seventh Avenue, New York, NY 10001, USA. Tel: +1(212) 584-4662.

DOI: http://dx.doi.org/10.1055/s-0031-1295686.

ISSN 1531-0043. 
THE MOLECULAR BIOLOGY OF OBESITY AS A RISK FACTOR FOR COLORECTAL CANCER

\section{Insulin and IGF-1}

Plasma insulin levels are elevated in the obese compared with the nonobese based on disruption in normal energy utilization and a need for higher insulin concentrations to carry out metabolic processes. This is true regardless of the diabetic status. ${ }^{1}$ Insulin then can stimulate production of insulin-like growth factor 1 (IGF-1), with higher levels of IGF-1 demonstrated in the obese compared with the nonobese. ${ }^{1}$ This occurs via the upregulation of growth hormone receptors in the liver, which then bind with secreted growth hormone, the main stimulus for IGF-1 production. ${ }^{2}$ Together, insulin and IGF-1 are thought to activate several signaling pathways associated with an elevated risk of oncogenesis.

What is the mechanism? First, IGF-1 on its own prevents apoptosis and facilitates cell cycle progression. ${ }^{1}$ Second, insulin may downregulate the production of insulin growth factor binding proteins 1 and 2 (IGFBP 1 and 2), which bind IGF-1 and inactivate it. ${ }^{2}$ IGF-1 receptors exist on both normal and colonic adenocarcinoma cells, and binding of IGF-1 results in cell cycle progression and inhibition of normal apoptosis in both normal and cancer cell lines. ${ }^{1}$ In addition, IGF-1 is capable of promoting angiogenesis and is overexpressed in transformed cells. ${ }^{2,3}$ Finally, there is a significant link between insulin/IGF-1 and the ras activation pathway. It is well known that colon cancer cells frequently overexpress ras proto-oncogene, which induces transformation from adenoma to invasive carcinoma. ${ }^{1}$ The activation of ras by farnesyl transferase occurs with the addition of a C15-prenyl group to its $\mathrm{C}$-terminal, which is promoted by insulin, and is essential for ras to travel to the plasma membrane of the cell to exert its transforming effects. $^{2}$

Acromegaly serves as a model for the effects of increased free IGF-1 concentrations, resulting in colon epithelial cell proliferation. In acromegaly, high growth hormone secretion leads to a significant increase in IGF1 secretion and an elevated production of IGF binding proteins. This ultimately produces an overall positive balance of circulating free IGF- $1 .{ }^{4}$ A meta-analysis of nine controlled studies examined the risk of developing colonic hyperplastic polyps, adenomas, and carcinomas in acromegalics and found significant risk elevation for all three types of lesions thereby supporting the theory of IGF-1 and carcinogenesis ${ }^{4}$

\section{Leptin}

As a hormone and a cytokine produced primarily by fat cells, leptin levels have been shown to be close to five times higher in obese as compared with nonobese sub- jects. ${ }^{5}$ Leptin functions primarily to induce a feeling of satiety as it binds to leptin receptors in the brain ${ }^{1}$; however, it has also been shown to stimulate two oncogenic pathways in colonic adenocarcinoma cell lines, MAPK and NF-кB, with resulting mitosis. ${ }^{1}$ Leptin has also been shown to inhibit apoptosis, induce angiogenesis, and promote cellular proliferation, similar to the effects of IGF- $1 .{ }^{1}$ In addition, leptin in vitro participates in the malignant behavior of cells by stimulating the invasive capacity of early neoplastic cells and increasing formation of lamellipodial structures important in cell motility. ${ }^{5}$ Leptin may interact with a variety of signaling pathways, including JAK2 tyrosine kinase, phosphoinositide kinase, mammalian target of rapamycin kinase, and protein kinase $\mathrm{C}$ to promote cellular proliferation, invasiveness, and oncogenesis. ${ }^{1}$

\section{Adipocytokines}

By poorly understood mechanisms, obesity causes lowgrade chronic inflammation through a multitude of inflammatory molecules known to be secreted by resident macrophages in adipose tissue. Adipocytokines are inflammatory molecules secreted by resident macrophages in adipose tissues. The mechanisms are yet to be elucidated, but these molecules appear to cause lowgrade chronic inflammation. Among them are such potent proinflammatory substances as TNF- $\alpha$, IL-6, monocyte chemoattractant protein-1, plasminogen activator inhibitor-1, adiponectin, leptin, and many others. ${ }^{6}$ In addition, excess adipose tissue acts as an independent endocrine organ, secreting fat-associated cytokines known as Adipocytokines, ${ }^{6}$ which can act to promote insulin resistance. ${ }^{6}$

As evidence for this low-grade chronic inflammatory state, the sedentary lifestyle associated with obesity is linked to elevated levels of $\mathrm{C}$-reactive protein. ${ }^{1}$ There are two pathways that may provide a link between this low-grade inflammatory state associated with obesity and colorectal cancer. The first is NF-кB, a "master switch" in the transcriptional regulation of $\sim 150$ genes that is known to directly activate oncogenes. It is activated by inflammatory cytokines such as IL-1 and TNF- $\alpha .{ }^{1}$ The second theory involves prostaglandin production. With decreased physical activity, as is often the case with obese subjects, prostaglandin levels in plasma fall, leading to a decrease in GI motility and stimulation of cell proliferation, potentially initiating the adenoma to carcinoma sequence. ${ }^{1}$ It has also been demonstrated that increased mucosal colonic prostaglandin production leads to more aggressive tumor behavior. ${ }^{7}$ In addition, high colonic mucosal $\mathrm{PGE}_{2}$ production is associated with colorectal polyps and cancer. ${ }^{7}$

Adiponectin, an adipocytokine, although not directly contributing to the inflammatory state, has been shown to be associated with dysplastic rectal aberrant 
crypt foci (potential clinical precursors of colorectal adenoma and carcinoma) in a clinical cohort. ${ }^{8}$ These lesions are also associated with mutations of the $K$-ras proto-oncogene in up to 50 to $60 \%$ of subjects. ${ }^{8}$ In vitro, adipocytes have been proven to induce colorectal cancer cell proliferation, a pathway in part regulated by leptin, but not completely dependent on it. ${ }^{9}$

\section{Sex Hormones}

Premenopausal women have high estrogen levels independent of body size. It is hypothesized that estrogen may upregulate IGF-1 and insulin receptors, with estrogen, IGF-1, and insulin competing for these receptors. The net effect of estrogen is protective. ${ }^{10}$ In the obese population, insulin production is increased, and with insulin receptors also upregulated, the estrogen protection is weakened, and oncogenesis may be favored. ${ }^{10}$ Androgen concentrations may have the same effect, though there are far fewer studies of this effect. Androgens, as precursors of estrogens, are also overproduced by the adipose tissue. However, androgens can activate IGF receptors unlike estrogens. In the end, the observed effect in population studies is that of increased insulin resistance, a common pathway. ${ }^{10}$

\section{A Special Risk Factor: Type 2 Diabetes Mellitus}

Any discussion of obesity and colorectal cancer would be incomplete without special mention of the diabetic population. According to estimates, most adults with type-2 diabetes (DM2) are overweight or obese. A recent study of 21,205 adults from California examined the prevalence of DM 2 across different body mass index (BMI) categories, and found that the prevalence of adults with diabetes increased with increasing weight, from $8 \%$ for normal weight individuals to $43 \%$ for individuals with $\mathrm{BMI} \geq 40 .{ }^{11}$ Almost a quarter of these diabetics had poor glycemic control.

This is important because elevated glycohemoglobin levels have been proven to be an independent predictor of early onset of CRC, an increased incidence of right-sided CRC, a more advanced stage at the time of presentation, and a reduced 5-year survival. ${ }^{12}$ Multiple studies link DM2 with increased risk of proximal and distal colon cancers as well as rectal cancers. In addition, populations on insulin therapy have been shown to have a threefold higher risk of colorectal cancer. ${ }^{13}$ The mechanisms responsible seem to be early hyperinsulinemia, enhanced by increased insulin resistance, and increased free IGF-1, leading to cellular proliferation, inhibition of apoptosis, and carcinogenesis. ${ }^{13}$ Multiple molecular pathways are likely involved and interact, including p-21-activated protein kinase, mammalian target of rapamycin, $\beta$-catenin, $W n t$, and glucagon-like peptide- $1 .{ }^{13}$

\section{The Crucial Effect of Exercise}

Exercise has been shown to decrease the incidence of colorectal cancer even in obese populations. In a large prospective cohort of Swedish men followed for 7.1 years, a significantly lower risk of colorectal cancer was documented for all BMI groups for men doing an hour of leisure time physical activity daily. ${ }^{14}$ Another large prospective study of men and women over a 7-year period noted a $55 \%$ decrease in risk of colon cancer in those who walked and did one other physical activity for $\geq 7$ hours/ week. The same study noted a decrease in rectal cancer risk of $30 \%$ in participants who did any physical activity versus none. ${ }^{15}$ In a Japanese case-control study of 833 men and women, authors found protective effects of either job-related or non-job related physical activity for both distal colon and rectum cancer in men and distal colon in women. ${ }^{16} \mathrm{~A}$ recent meta-analysis of 14 studies examining the relationship between colorectal cancer and physical activity found a $20 \%$ risk reduction for men and a $14 \%$ risk reduction for women for colon cancer, with a linear dose-response relationship. Interestingly, this meta-analysis did not demonstrate any risk reduction for rectal cancer. ${ }^{17}$

Why does exercise help in reducing the risk of colorectal cancer? It seems that physical activity shortens intestinal transit time, thus reducing the contact time between the colorectal epithelium and neoplasia promoting dietary constituents. Exercise also enhances immune function; increases prostaglandin $\mathrm{F}$ and decreases prostaglandin $\mathrm{E}_{2}$; maintains insulin sensitivity; lowers the activity of insulin-like growth factor, sex hormones, and bile acid secretion; and decreases adiposity. ${ }^{16}$

\section{WHAT IS THE RISK OF COLORECTAL CANCER ASSOCIATED WITH OBESITY?}

\section{Measures of Obesity}

Existing literature leaves no doubt as to the existence of the positive relationship between colorectal cancer and obesity. However, prior to exploring the data, it is important to understand that there are different definitions of obesity and these variations affect the conclusions reached in various studies. The most common measure of obesity used in the literature is body mass index (BMI; expressed in $\mathrm{kg} / \mathrm{m}^{2}$ ). BMI is used as a surrogate measure of obesity, based on the fact that it takes into account height and weight. However, it fails to distinguish between fat and lean body mass, which vary significantly according to gender, age, ethnicity, and even geographic locale of the same ethnic group. ${ }^{18-21}$ BMI also does not take into account the distribution of weight (abdominal, subcutaneous), and there is substantial evidence that the World Health Organization (WHO) cutoffs for the definitions of obesity ${ }^{22}$ should 
be redefined for certain ethnic groups, most notably Asians.

Despite its shortcomings, BMI has been a widely applied tool for estimation of obesity in the colorectal literature due to ease of use. Other measures of obesity are waist circumference (WC) and waist to hip ratio (WHR). These measures have also been widely implemented, primarily for specific measurement of abdominal obesity, which has a higher association with overall mortality and cardiovascular disease than overall obesity. However, WC and WHR cutoffs for abdominal obesity have been mostly studied in European populations and are poorly validated in most other ethnicities with trends for lower cutoffs suggested for Asian populations. ${ }^{23}$ As a final measure, some have used visceral fat accumulation as directly measured by magnetic resonance imaging or computed tomography. ${ }^{24,25}$ This measure too, however, shows different trends across genders and ethnicities ${ }^{26}$ with no well-documented universal cutoffs.

The evidence presented in this article regarding the link of colorectal cancer and obesity thus has to be viewed in the light of flawed definitions of obesity and large variations of population composition in the cited studies. Having stated this and despite these variations, it is worthwhile to note that the International Agency for Research into Cancer (IARC) recognized colorectal cancer as an obesity-related cancer in 2001, and in 2007 the World Cancer Research Fund (WRCF) concluded that existing evidence is sufficient to state that body fat is a cause of colorectal cancer. ${ }^{17}$ When examining results from published literature, it is worthwhile to divide the obese population by gender and diabetic status to achieve maximal clarity due to wide variations in results.

\section{Men}

Table 1 summarizes the most recent literature on the risk of colorectal cancer with increasing weight in men. ${ }^{17,27-}$ 32 Unfortunately, the specific studies use heterogenous cutoffs for absolute measures of weight, weight increase, and BMI, thus there is no single risk increase fraction that can be derived. However, the general trend is clear for colon cancer. Risk increases in a linear fashion with adult weight gain, and there appears to be an increased risk for proximal cancers, and possibly for distal colon cancers as well. Waist to hip ratio seems to be a stronger predictor than $\mathrm{BMI}$ alone in several studies.

For rectal cancer, there is less data, and results are less certain. Several studies reported increased rates of rectal cancer with obesity, though the association is weaker than that of obesity with colon cancer.

The relative risk of colon cancer rises with increasing weight gain in men; several studies provide very similar numbers for the actual risk increase. This can be explained by male distribution of adipose tissue: primar- ily central and abdominal, leading to more insulin resistance than in the same BMI woman. The most conclusive study to date is a meta-analysis that included 28 studies. Results were divided by gender and cancer location, and there was a separate analysis of potential confounding factors. ${ }^{17}$ This study used a BMI increase of $5 \mathrm{~kg} / \mathrm{m}^{2}$ as a measure of risk. Because BMI has many limitations, so does any analysis based on it. It is important to keep in mind that the conclusions reached in an Australian study showing that the association of BMI with colon cancer becomes insignificant if one controls for waist circumference and waist-hip ratio, whereas the significance remained if one controls for BMI while measuring colon cancer risk associated with waist circumference and waist-hip ratio. ${ }^{27}$

\section{Women}

Table 2 summarizes the most recent literature on the link between obesity and the risk of colorectal cancer in women. ${ }^{17,27,29-33}$ Generally, the association between obesity and colorectal cancer is much weaker in women than men, and any association between obesity and rectal cancer is questionable and ungrounded in most studies. However, it is not to be ignored, and it appears that the link of obesity to rectal cancer is strengthened significantly by family history. The weaker association of colon and rectal cancers with female obesity can be explained by different body fat distribution, higher insulin sensitivity, protective effects of estrogen, and difference in adipocytokines. Men and women of the same BMI have vastly different body fat proportion and distribution. Women have higher peripheral adiposity whereas men have more lean mass but more visceral and hepatic adipose tissue. Given hormonal differences and body composition differences, men have higher insulin resistance than women. ${ }^{34}$

In addition, the association between female gender, obesity, and colon cancer was stronger when waisthip ratio and waist circumference were used as surrogate measures of obesity instead of BMI. There was only weak association in North American studies for rectal cancer and no association in European or Asian studies.

In regards to hormonal status, it is difficult to draw conclusions. Adequately powered large studies often lack data as far as duration of menopause and presence/absence of hormone replacement therapy.

\section{Type 2 Diabetes Mellitus (DM2)}

There are a growing number of type 2 diabetics as the obesity epidemic expands. Considering that more than $40 \%$ of those with BMI $>40$ are diabetic, ${ }^{11}$ the relationship between DM2 and colorectal cancer is important to understand in counseling and evaluating high-risk 
Table 1 Summary of Recent Studies Addressing Obesity and Risk of Colorectal Cancer in Men

\begin{tabular}{|c|c|c|c|c|c|}
\hline & $\begin{array}{l}\text { Country; } \\
\text { Type of Study; } \\
\text { Participants }\end{array}$ & Follow-up & $\begin{array}{l}\text { Method } \\
\text { Weight } \\
\text { Obtained }\end{array}$ & $\begin{array}{l}\text { Risk Colon } \\
\text { Cancer }\end{array}$ & $\begin{array}{l}\text { Risk } \\
\text { Rectal } \\
\text { Cancer }\end{array}$ \\
\hline $\begin{array}{l}\text { Bassett } \\
\text { et al, } 2010^{27}\end{array}$ & $\begin{array}{l}\text { Australia; prospective } \\
\text { cohort; } 16,188 \text { men, } \\
\text { age } 40-69 \text { at entry } \\
\text { mostly }\end{array}$ & $\begin{array}{l}14 \text { Years } \\
\text { on average }\end{array}$ & $\begin{array}{l}\text { Measured height } \\
\text { and weight at } \\
\text { study entry; } \\
\text { recall for remote } \\
\text { data }\end{array}$ & $\begin{array}{l}\mathrm{BMI} \geq 30 \text { at study entry: } \mathrm{HR} \text {, } \\
1.51 ; 95 \% \mathrm{Cl}, 1.00-2.28 \\
\text { Adult weight gain } \geq 20 \mathrm{~kg} \text { : } \\
\mathrm{HR}, 1.47 ; 95 \% \mathrm{Cl}, 0.94-2.31 \\
\text { Proximal colon } \mathrm{CA} \text { among } \\
\text { men who gained } \geq 20 \mathrm{~kg} \text { : } \\
\mathrm{HR}, 2.12 ; 95 \% \mathrm{Cl}, 1.10-4.10 \\
\text { Risk of CA fit linear model } \\
\text { and increased } 11 \% \text { every } \\
5 \mathrm{~kg} \text { of weight gain. }\end{array}$ & $\begin{array}{l}\text { Not } \\
\text { examined }\end{array}$ \\
\hline $\begin{array}{l}\text { Thygesen } \\
\text { et al, } 2008^{28}\end{array}$ & $\begin{array}{l}\text { U.S.; prospective } \\
\text { cohort; } 46,349 \text { male } \\
\text { health professionals, } \\
\text { age } 40-75 \text { at } \\
\text { enrollment }\end{array}$ & $\begin{array}{l}18 \text { Years } \\
\text { or until } \\
\text { endpoint } \\
\text { (diagnosis } \\
\text { of CRC or } \\
\text { death) }\end{array}$ & $\begin{array}{l}\text { Self-reported } \\
\text { height and } \\
\text { weight* }\end{array}$ & $\begin{array}{l}\mathrm{BMI} 30.1-35 \text { and }>35 \text { at } \\
\text { study entry: } \mathrm{HR}, 2.29 ; 95 \% \\
\mathrm{Cl}, 1.58-3.31 \text { and } 1.23-4.26 \text {, } \\
\text { respectively Proximal colon } \\
\mathrm{CA} \text { among men who gained } \\
4.54 \mathrm{~kg} \text { over } 2 \text { years: HR, } \\
1.39 ; 95 \% \mathrm{Cl}, 1.14-1.68 \\
\text { Risk of CA fit time lag linear } \\
\text { model and increased } 14 \% \\
\text { for every } 4.54 \mathrm{~kg} \text { of gain. }\end{array}$ & $\begin{array}{l}\text { Not } \\
\text { examined }\end{array}$ \\
\hline $\begin{array}{l}\text { Pischon } \\
\text { et al, } 2006^{29} \\
\text { (EPIC) }\end{array}$ & $\begin{array}{l}10 \text { European } \\
\text { countries; } 129,731 \\
\text { men, mean age } \\
51.7 \text { years }\end{array}$ & 6.1 Years & $\begin{array}{l}\text { Measured } \\
\text { height, weight, } \\
\text { WC, WHR }\end{array}$ & $\begin{array}{l}\text { Risk of CA fit linear model } \\
\text { and increased } 9 \% \text { every } 5 \mathrm{~kg} \\
\text { of weight gain. } \\
\text { WC: RR, } 1.39 ; 95 \% \mathrm{Cl} \text {, } \\
1.01-1.93 \text { ( } \geq 103 \mathrm{~cm} \text { vs } \\
<86 \mathrm{~cm} \text { ) } \\
\text { WHR (strongest } \\
\text { association): RR, } 1.51 ; 95 \% \\
\mathrm{Cl}, 1.06-2.15 \text { ( } \geq 0.99 \\
\text { vs } \leq 0.89 \text { ) } \\
\mathrm{BMI} \geq 29.4 \text { : RR, } 1.55 ; 95 \% \\
\mathrm{Cl}, 1.12-2.15\end{array}$ & $\begin{array}{l}\text { WHR: RR, } \\
1.93 ; 95 \% \mathrm{Cl} \text {, } \\
1.19-3.13 \text { for } \\
\text { ratio }>0.99 \text { vs } \\
<0.887\end{array}$ \\
\hline $\begin{array}{l}\text { Larsson and } \\
\text { Wolk, } 2007^{30}\end{array}$ & $\begin{array}{l}\text { Meta-analysis of } 31 \\
\text { prospective studies; } \\
12 \text { studies U.S., } 11 \\
\text { studies Europe, } 3 \\
\text { Australia, } 1 \text { Korea, } 4 \\
\text { Japan; 3,128,274 men }\end{array}$ & $\begin{array}{l}\text { Variable, } \\
\text { from } \\
4.8-30 \\
\text { years }\end{array}$ & $\begin{array}{l}17 / 31 \text { Studies } \\
\text { measured } \\
\text { weight and } \\
\text { height; the rest } \\
\text { relied on } \\
\text { self-report }\end{array}$ & $\begin{array}{l}\text { BMI increase of } 5 \text { units: } \\
30 \% \text { increased risk of } \\
\text { colon CA } \\
\text { BMI increase of } 5 \text { units: } \\
\text { Proximal CA RR, 1.29; 95\% } \\
\mathrm{Cl}, 1.17-1.42 \text {. Distal CA RR, } \\
1.35 ; 95 \% \mathrm{Cl}, 1.22-1.48 \text {. } \\
\text { WC RR, 1.33; WHR RR, } \\
1.43 \text {, significantly associated } \\
\text { with colon CA. } \\
{ }^{*} \text { Associations stronger in } \\
\text { U.S. studies compared with } \\
\text { European and Asian studies }\end{array}$ & $\begin{array}{l}\text { BMI increase } \\
\text { of } 5 \text { units: } 12- \\
16 \% \text { increased } \\
\text { risk of rectal } \\
\text { CA in } \\
\text { European and } \\
\text { Asian studies, } \\
1.09-1.15 \text { and } \\
1.05-1.28 \text {, } \\
\text { respectively. } \\
\text { WC is } \\
\text { significantly } \\
\text { associated } \\
\text { with rectal CA. }\end{array}$ \\
\hline
\end{tabular}


Table 1 (Continued)

\begin{tabular}{|c|c|c|c|c|c|}
\hline & $\begin{array}{l}\text { Country; } \\
\text { Type of Study; } \\
\text { Participants }\end{array}$ & Follow-up & $\begin{array}{l}\text { Method } \\
\text { Weight } \\
\text { Obtained }\end{array}$ & $\begin{array}{l}\text { Risk Colon } \\
\text { Cancer }\end{array}$ & $\begin{array}{l}\text { Risk } \\
\text { Rectal } \\
\text { Cancer }\end{array}$ \\
\hline $\begin{array}{l}\text { Dai et al, } \\
2007^{31}\end{array}$ & $\begin{array}{l}\text { Meta-analysis of } 15 \\
\text { prospective cohort } \\
\text { studies; } 6 \text { studies } \\
\text { U.S., } 5 \text { in Europe, } 2 \text { in } \\
\text { Australia, } 1 \text { in } \\
\text { Canada, } 1 \text { in Japan }\end{array}$ & Variable & $\begin{array}{l}\text { 7/17 Studies } \\
\text { measured } \\
\text { weight, height, } \\
\text { WC, and WHR }\end{array}$ & $\begin{array}{l}\text { BMI (highest vs lowest } \\
\text { quantile): RR, 1.59; } 95 \% \mathrm{Cl} \text {, } \\
\text { 1.35-1.86 } \\
\text { WC (highest vs lowest } \\
\text { quantile): RR, 1.68; } 95 \% \mathrm{Cl} \text {, } \\
\text { 1.36-2.08 } \\
\text { WHR (highest vs lowest } \\
\text { quantile): RR, 1.91; } 95 \% \mathrm{Cl} \text {, } \\
\text { 1.46-2.49 }\end{array}$ & $\begin{array}{l}\text { BMI: Trend } \\
\text { up, no } \\
\text { significance. } \\
\text { WC: Trend } \\
\text { up, no } \\
\text { significance }\end{array}$ \\
\hline $\begin{array}{l}\text { Moghaddam } \\
\text { et al, } 2007^{\dagger 32}\end{array}$ & $\begin{array}{l}\text { Meta-analysis of } 31 \\
\text { observational studies } \\
\text { ( } 23 \text { cohort and } 8 \text { case- } \\
\text { control); } 15 \text { studies in } \\
\text { U.S., } 7 \text { in Europe, } 5 \\
\text { in Australia, } 2 \text { in } \\
\text { Canada, } 2 \text { in Japan, } \\
\text { and } 1 \text { in Korea; } \\
70,906 \text { cases of } \\
\text { colorectal cancer }\end{array}$ & Variable & Not reported & $\begin{array}{l}25<\mathrm{BMI}<29.9 \text { : RR, 1.23; } \\
95 \% \mathrm{Cl}, 1.11-1.36 \\
\mathrm{BMI}>30: \mathrm{RR}, 1.53 ; 95 \% \mathrm{Cl}, \\
\text { 1.33-1.75, after correction } \\
\text { for bias. } \\
\text { Pooled corrected (men + } \\
\text { women) risk of colorectal } \\
\mathrm{CA} \text { increases } 6 \% \text { for every } \\
2 \text { increment increase in } \mathrm{BMI} \text {. }\end{array}$ & $\begin{array}{l}\text { BMI > 30: RR, } \\
1.27 ; 95 \% \mathrm{Cl} \text {, } \\
1.17-1.37, \\
\text { after correction } \\
\text { for bias. } \\
\text { Pooled (men + } \\
\text { women) risk of } \\
\text { colorectal CA } \\
\text { increases } 4 \% \\
\text { for every } 2 \mathrm{~cm} \\
\text { increase in } \\
\text { WC. }\end{array}$ \\
\hline $\begin{array}{l}\text { Harriss } \\
\text { et al, } 2009^{17}\end{array}$ & $\begin{array}{l}\text { Meta-analysis of } 28 \\
\text { studies; } 3 \text { are case- } \\
\text { controls, } 25 \text { cohorts; } \\
13 \text { studies in U.S./ } \\
\text { Canada, } 9 \text { in Europe, } \\
3 \text { in Australia, and } 4 \text { in } \\
\text { Japan/Korea }\end{array}$ & Variable & $\begin{array}{l}\text { 16/28 Measured } \\
\text { weight, height }\end{array}$ & $\begin{array}{l}\text { BMI increase of } 5 \text { units: RR, } \\
1.24 ; 95 \% \mathrm{Cl}, 1.20-1.28 \\
\text { BMI increase of } 5 \text { units: } \\
\text { Distal colon RR, } 1.28 ; 95 \% \\
\mathrm{Cl}, 1.18-1.39 \\
\text { Associations are stronger in } \\
\text { North America and Pacific } \\
\text { and when controlled for } \\
\text { physical activity. }\end{array}$ & $\begin{array}{l}\text { BMI increase } \\
\text { of } 5 \text { units: } \\
\mathrm{RR}, 1.09 \text {; } \\
95 \% \mathrm{Cl}, 1.06- \\
1.12 \\
\text { Associations } \\
\text { are stronger } \\
\text { when } \\
\text { adjusted for } \\
\text { family history } \\
\text { and physical } \\
\text { activity. }\end{array}$ \\
\hline
\end{tabular}

$\mathrm{BMI}$, body mass index; $\mathrm{CA}$, cancer; $\mathrm{Cl}$, confidence interval; $\mathrm{CRC}$, colorectal cancer; HR, hazards ratio; RR, relative risk; WC, waist circumference; WHR, waist-to-hip ratio.

* Self-reported past body weight has $64-95 \%$ correlation with actual weight. ${ }^{27}$

'This study reported significant publication bias and heterogeneity among individual studies.

patients. In most studies, a 30 to $40 \%$ risk of colorectal cancer is imparted by the diagnosis of DM2. ${ }^{35}$ This risk is even higher when there is a family history, and there appears to be increased risk for proximal colon cancers. ${ }^{35}$

The vast majority of large prospective cohorts examining the association between DM2 and colorectal cancer were done in the late 1990s and early 2000s. A meta-analysis of 15 studies, done by the same Swedish group that analyzed the relationship between obesity and colorectal cancer in 2007, provides a summary of large studies published between 1985 to $2005 .{ }^{36}$ Although type 1 and 2 diabetics are often combined, especially in early studies, and only several studies controlled for BMI and physical activity, the authors did subset analyses to ensure that physical activity, BMI, and inclusion of type 1 diabetics in early studies did not impact results, and found no significant differences. The analyzed cohort consisted of over 2.5 million subjects. The authors concluded that women (relative risk [RR], 1.33; 95\% confidence interval [CI], 1.23-1.44) and men (RR, 1.29; 95\% CI, 1.15-1.44) had a similar increase in colorectal cancer risk associated with diabetes. The risk was slightly higher in Europe than in the United States. There were no significant differences in subsite preponderance in diabetics: proximal colon RR, 1.64 (95\% CI, 1.31-2.05), distal colon RR, 1.49 (95\% CI,1.12-1.99), and rectum 
Table 2 Summary of Recent Studies Addressing Obesity and Risk of Colorectal Cancer in Women

\begin{tabular}{|c|c|c|c|c|c|}
\hline & $\begin{array}{l}\text { Country; } \\
\text { Type of Study; } \\
\text { Participants }\end{array}$ & Follow-up & $\begin{array}{l}\text { Method } \\
\text { Weight } \\
\text { Obtained }\end{array}$ & $\begin{array}{l}\text { Risk Colon } \\
\text { Cancer }\end{array}$ & $\begin{array}{l}\text { Risk Rectal } \\
\text { Cancer }\end{array}$ \\
\hline $\begin{array}{l}\text { Bassett } \\
\text { et al, } 2010^{27}\end{array}$ & $\begin{array}{l}\text { Australia; prospective } \\
\text { cohort; } 23,438 \text { women, } \\
\text { age } 40-69 \text { years at entry } \\
\text { mostly }\end{array}$ & $\begin{array}{l}14 \text { Years } \\
\text { on average }\end{array}$ & $\begin{array}{l}\text { Measured height } \\
\text { and weight at } \\
\text { study entry; } \\
\text { recall for remote } \\
\text { data }\end{array}$ & $\begin{array}{l}\text { No association found } \\
\text { between BMI at age } 18 \text { or at } \\
\text { study entry and risk of colon } \\
\text { CA; no association with } \\
\text { adult weight gain and colon } \\
\text { CA }\end{array}$ & Not examined \\
\hline $\begin{array}{l}\text { Oxentenko } \\
\text { et al, } 2010^{33}\end{array}$ & $\begin{array}{l}\text { U.S. lowa Women's } \\
\text { Health Study; } \\
\text { prospective cohort; } \\
36,941 \text { women, age } \\
55-69 \text { years at study } \\
\text { entry }\end{array}$ & $\begin{array}{l}19 \text { Years } \\
\text { or until } \\
\text { endpoint } \\
\text { (diagnosis } \\
\text { of CRC) }\end{array}$ & $\begin{array}{l}\text { Self-reported* } \\
\text { height and } \\
\text { weight, estrogen, } \\
\text { and OCP use }\end{array}$ & $\begin{array}{l}\mathrm{BMI} 30-34.9 \text { : RR, 1.31; 95\% } \\
\mathrm{Cl}, 1.12-1.54 \\
\mathrm{BMI}>40: \mathrm{RR}, 1.56 ; 95 \% \mathrm{Cl} \text {, } \\
\text { 1.10-2.22 } \\
\mathrm{BMI}>40 \text { : Distal colon CA } \\
\text { RR, 1.86; 95\% Cl, 1.14- } \\
\text { 3.05, but no association with } \\
\text { proximal CA. } \\
\text { Peak: Postmenopausal BMI } \\
>40 \text { vs normal: RR, 1.52; } \\
95 \% \text { Cl, 1.10-2.11 } \\
\text { Peak: Premenopausal. } \\
\text { BMI >40 vs normal: No } \\
\text { significance }\end{array}$ & $\begin{array}{l}\text { Rectal CAs were } \\
\text { included in the } \\
\text { analysis of distal } \\
\text { CAs. }\end{array}$ \\
\hline $\begin{array}{l}\text { Pischon } \\
\text { et al, } 2006^{29} \\
\text { (EPIC) }\end{array}$ & $\begin{array}{l}10 \text { European countries; } \\
238,546 \text { women, mean } \\
\text { age } 51.1 \text { years }\end{array}$ & 6.1 Years & $\begin{array}{l}\text { Measured } \\
\text { height, weight, } \\
\text { WC, HR }\end{array}$ & $\begin{array}{l}\text { WC: RR, 1.48; } 95 \% \mathrm{Cl} \text {, } \\
1.08-2.03, \text { for }>89 \mathrm{~cm} \\
\text { Vs }<70.2 \mathrm{~cm} \\
\text { WHR (strongest association): } \\
\text { RR, } 1.52,95 \% \mathrm{Cl}, 1.12-2.05 \\
\text { No association between } \\
\text { weight at entry, weight gain, } \\
\text { or BMI with colon CA. } \\
\text { Postm enopausal women had } \\
\text { same results as all women. } \\
\text { HRT alone was not related to } \\
\text { risk of colon CA in post } \\
\text { menopausal women. }\end{array}$ & $\begin{array}{l}\text { No anthropometric } \\
\text { measurements } \\
\text { were related to } \\
\text { rectal CA. }\end{array}$ \\
\hline $\begin{array}{l}\text { Larsson and } \\
\text { Wolk, } 2007^{30}\end{array}$ & $\begin{array}{l}\text { Meta-analysis of } 31 \\
\text { prospective studies; } \\
12 \text { studies U.S., } \\
11 \text { studies Europe, } \\
3 \text { Australia, } 1 \text { Korea, } \\
4 \text { Japan; 2,419,875 } \\
\text { women }\end{array}$ & $\begin{array}{l}\text { Variable, } \\
\text { from } 4.8- \\
30 \text { years }\end{array}$ & $\begin{array}{l}17 / 31 \text { Studies } \\
\text { measured } \\
\text { weight and } \\
\text { height; the rest } \\
\text { relied on } \\
\text { self-report }\end{array}$ & $\begin{array}{l}\text { BMI increase of } 5 \text { units: } \\
12 \% \text { increased risk of } \\
\text { colon CA } \\
\text { BMI increase of } 5 \text { units: } \\
\text { Distal CA RR, } 1.14 ; 95 \% \mathrm{Cl} \text {, } \\
1.01-1.28 \\
\text { WC (RR, 1.16) and WHR } \\
\text { (RR, 1.20) associated with } \\
\text { colon CA, but weaker } \\
\text { association than in men. } \\
{ }^{*} \text { Associations stronger in } \\
\text { U.S. studies compared with } \\
\text { European and Asian studies. }\end{array}$ & $\begin{array}{l}\text { BMI increase of } \\
5 \text { units: No } \\
\text { significant effect } \\
\text { in women overall }\end{array}$ \\
\hline
\end{tabular}


Table 2 (Continued)

\begin{tabular}{|c|c|c|c|c|c|}
\hline & $\begin{array}{l}\text { Country; } \\
\text { Type of Study; } \\
\text { Participants }\end{array}$ & Follow-up & $\begin{array}{l}\text { Method } \\
\text { Weight } \\
\text { Obtained }\end{array}$ & $\begin{array}{l}\text { Risk Colon } \\
\text { Cancer }\end{array}$ & $\begin{array}{l}\text { Risk Rectal } \\
\text { Cancer }\end{array}$ \\
\hline Dai et al, $2007^{31}$ & $\begin{array}{l}\text { Meta-analysis of } 15 \\
\text { prospective cohort } \\
\text { studies; } 6 \text { studies U.S., } \\
5 \text { in Europe, } 2 \text { in } \\
\text { Australia, } 1 \text { in Canada, } \\
1 \text { in Japan }\end{array}$ & Variable & $\begin{array}{l}\text { 7/17 Studies } \\
\text { measured } \\
\text { weight, height, } \\
\text { WC, and WHR }\end{array}$ & $\begin{array}{l}\text { BMI (highest vs lowest } \\
\text { quantile): RR, 1.22; } 95 \% \mathrm{Cl} \text {, } \\
\text { 1.08-1.39 } \\
\text { WC (highest vs lowest } \\
\text { quantile): RR, 1.48; } 95 \% \mathrm{Cl} \text {, } \\
1.19-1.84 \\
\text { WHR (highest vs lowest } \\
\text { quantile): RR, } 1.4995 \% \mathrm{Cl} \text {, } \\
1.23-1.81\end{array}$ & $\begin{array}{l}\text { BMI: No } \\
\text { significant } \\
\text { association } \\
\text { WC: No significant } \\
\text { association } \\
\text { WHR (highest vs } \\
\text { lowest quantile): } \\
\text { No significant } \\
\text { association }\end{array}$ \\
\hline $\begin{array}{l}\text { Moghaddam } \\
\text { et al, } 2007^{\dagger 32}\end{array}$ & $\begin{array}{l}\text { Meta-analysis of } 31 \\
\text { observational studies } \\
\text { ( } 23 \text { cohort and } 8 \text { case- } \\
\text { control); } 15 \text { studies in } \\
\text { US, } 7 \text { in Europe, } 5 \text { in } \\
\text { Australia, } 2 \text { in Canada, } \\
2 \text { in Japan, and } 1 \text { in } \\
\text { Korea; } 70,906 \text { cases } \\
\text { of CRC }\end{array}$ & Variable & Not reported & $\begin{array}{l}\text { BMI >30: No statistical } \\
\text { significance } \\
\text { Pooled corrected (men + } \\
\text { women) risk of CRC } \\
\text { increases } 6 \% \text { for every } 2 \\
\text { increment increase in BMI. }\end{array}$ & $\begin{array}{l}\text { BMI > 30: No } \\
\text { statistical } \\
\text { significance } \\
\text { Pooled (men + } \\
\text { women) risk of } \\
\text { CRC increases } \\
4 \% \text { for every } \\
2 \mathrm{~cm} \text { increase } \\
\text { in WC. }\end{array}$ \\
\hline $\begin{array}{l}\text { Harriss } \\
\text { et al, } 2009^{17}\end{array}$ & $\begin{array}{l}\text { Meta-analysis of } 28 \\
\text { studies; } 3 \text { are case- } \\
\text { controls, } 25 \text { cohorts; } \\
13 \text { studies in U.S./ } \\
\text { Canada, } 9 \text { in Europe, } \\
3 \text { in Australia, and } \\
4 \text { in Japan/Korea }\end{array}$ & Variable & $\begin{array}{l}\text { 16/28 Measured } \\
\text { weight, height }\end{array}$ & $\begin{array}{l}\text { BMI increase of } 5 \text { units: RR, } \\
1.09 ; 95 \% \mathrm{Cl}, 1.04-1.14 \\
\text { No site-specific significance } \\
\text { Associations are stronger in } \\
\text { North America and when } \\
\text { controlled for physical activity. }\end{array}$ & $\begin{array}{l}\text { BMI increase } \\
\text { of } 5 \text { units: No } \\
\text { significance } \\
\text { Associations are } \\
\text { stronger when } \\
\text { BMl self-reported, } \\
\text { adjusted for } \\
\text { family history, } \\
\text { and physical } \\
\text { activity. }\end{array}$ \\
\hline
\end{tabular}

BMI, body mass index; $\mathrm{CA}$, cancer; $\mathrm{Cl}$, confidence interval; $\mathrm{CRC}$, colorectal cancer; HR, hazards ratio; HRT, hormone replacement therapy; $\mathrm{OCP}$, oral contraceptive pill; RR, relative risk; WC, waist circumference; WHR, waist-to-hip ratio.

* Self-reported past body weight has $64-95 \%$ correlation with actual weight. ${ }^{27}$

${ }^{\dagger}$ This study reported significant publication bias and heterogeneity among individual studies.

RR,1.33 (95\% CI, 1.14-1.54). ${ }^{36}$ There was no heterogeneity among these studies. In contrast, the same group also showed that diabetes was associated with increased colorectal cancer mortality (RR, 1.26; 95\% CI, 1.05$1.5)$, although there was significant heterogeneity between studies on this matter. Other studies also report different risks associated with proximal versus distal subsites, and there is no definitive conclusion at this point regarding colorectal cancer subsite predominance in diabetics. ${ }^{37}$

The strongest study to date analyzing insulin therapy for DM2 as a risk factor for colorectal cancer was published in 2004 by Yang et al, retrospectively looking at 25,000 British subjects. ${ }^{38}$ These authors noted a doubling of overall risk in developing colorectal cancer among their DM2 population. More specifically, patients that were on insulin therapy for at least 3 years had three times the risk of noninsulin-dependent dia- betics of developing colorectal cancer (odds ratio [OR], 3.4; $95 \% \mathrm{CI}, 1.5-7.7 ; P=.004)$. When insulin therapy was examined as a continuous variable, a multivariate analysis showed a risk increase of $21 \%$ of colorectal cancer per each year of treatment with insulin $(\mathrm{OR}$, 1.21; 95\% CI, 1.03-1.42; $P=.02) .{ }^{38}$ A major limitation of this study, however, was that data regarding glycemic control and the exact insulin regimen was not analyzed. It has been previously shown that high glycohemoglobin $(\mathrm{HbA} 1 \mathrm{c}>7.5 \%)$ is associated with younger age at presentation, more advanced stage, and poorer 5-year survival. $^{12}$

It can be concluded that diabetes is at least a moderate risk factor for colorectal cancer, and in combination with other factors such as obesity and insulin therapy, the risk is much increased. This is most likely due to early hyperinsulinemia and the subsequent rise in IGF-1 associated with DM2, which creates a favorable 
environment for transformed cell proliferation and lack of apoptosis. ${ }^{2,35}$ Current screening guidelines do not recommend screening diabetics or obese patients earlier, though they do note that obese patients are at a greater risk. $^{39}$ This may well change in the near future. Although population studies from Europe warrant such a change, there is a need for American population studies that focus on indices of glycemic control, type of diabetic regimen, and onset of diabetes to establish who is at greatest risk and when screening should be offered before we suggest that cost-effective changes to the current screening guidelines should be implemented.

\section{Obesity and Colorectal Surgery Outcomes}

In understanding the influence of obesity on colorectal surgery outcomes, there are several confounding variables that make reaching firm conclusions, based on the available data, difficult. Much of the data comes from retrospective cohort or case-control trials that are sometimes underpowered and often contradictory. In addition, many trials include patients treated with surgery alone along with those treated with various adjuvant or neoadjuvant chemotherapeutic or chemoradiation regimens. Finally, obesity, in itself a continuous variable, is defined in most of these trials as a cutoff at BMI $\geq 25$ or 30 , and no other surrogate definitions of obesity are consistently used. It is thus quite possible that the outcomes incurred by the very obese $(\mathrm{BMI}>40)$ are underestimated because this population is "hidden" inside the general obese population.

\section{Tumor Grade, Lymph Node Status, and Lymph Node Retrieval}

A retrospective Spanish study examined 369 patients with colon and rectal cancers and found significant associations with obesity. The vast majority of both colon and rectal cancers were adenocarcinomas. Of the study population, $32.1 \%$ were overweight and $16.6 \%$ were obese by BMI definition $\left(\geq 30 \mathrm{~kg} / \mathrm{m}^{2}\right)$. BMI was calculated preoperatively from measured height and weight. Bivariate analysis for colon cancer cases determined worse tumor differentiation in patients with BMI $>25$ versus those with BMI $<25 \mathrm{~kg} / \mathrm{m}^{2}(P=.011)$ and significantly more affected lymph nodes in those with $\mathrm{BMI} \geq 30$ versus those with BMI $<30 \mathrm{~kg} / \mathrm{m}^{2}(P=.043)$. Bivariate analysis for rectal cancer cases revealed higher TNM stage $(P=.023)$ with increasing BMI and more affected lymph nodes in those with BMI $>25$ versus those with lower BMI $(P=.041){ }^{40}$ This study did not subdivide by gender, all cases were done via an open approach, and there was no mention of neoadjuvant chemoradiation in any of the patients. There was no difference in lymph node retrieval in obese compared with normal-weight subjects for colon or rectal cancer.
Another retrospective study of 4,381 stage II and III colon carcinoma patients found that obese patients were more likely to have more than three metastatic lymph nodes compared with normal-weight patients at the time of resection (28\% vs. $22 \%, P=.017) .{ }^{41}$ An Irish retrospective analysis examining 414 patients (273 with colon cancer, 141 with rectal cancer) found no differences in lymph node retrieval or tumor differentiation between the obese cohort $(\mathrm{BMI}>30)$ and nonobese cohort. ${ }^{42}$ However, they found that for obese men with either colon or rectal cancer, there were significantly more cases with more than four positive lymph nodes involved $(P=.012)$, and $4+$ lymph node involvement was significantly more common in the obese cohort of patients with colon cancer only $(P=.017)$. This study was complicated by the fact that 77 patients received neoadjuvant chemotherapy and radiation therapy, 179 had adjuvant chemotherapy, and 35 had adjuvant radiotherapy. Thus, there is a distinct possibility that the outcomes may have been influenced by administration of neoadjuvant therapy to some patients in this study. There was no multivariate analysis to control for this factor. A retrospective observational study of 1,053 patients with stage III colon cancer enrolled in a randomized trial of adjuvant chemotherapy after cancer resection reported no difference in lymph node retrieval across patient-reported $\mathrm{BMI}$ ranges $(P=.27) .{ }^{43}$

Specifically addressing the issue of lymph node harvest, Linebarger et al retrospectively reviewed medical records of 401 patients who underwent colon cancer resection. ${ }^{44}$ There was no difference in lymph node retrieval among all BMI groups, even in BMI $>40$ $(P=.321)$. In this study, a laparoscopic approach was used in $26 \%$ of cases, $5 \%$ of laparoscopic cases were converted to open, $68 \%$ of cases were open, and a few cases were approached with a hand-assisted technique. Factors found to significantly impact lymph node harvest were stage of cancer, left-sided location, and the pathology technician. Acetic acid was used after manual dissection to reveal additional lymph nodes, a technique that greatly aids in identifying otherwise poorly visualized nodes. A smaller retrospective review of 191 colon cancer patients stratified by $\mathrm{BMI}<30, \mathrm{BMI} \geq 30$, and $\mathrm{BMI} \geq 40$ revealed no differences in lymph node harvest. $^{45}$

Lymph node retrieval during rectal cancer surgery yields similar results. A French retrospective review of 210 mesorectal excisions, where most patients received neoadjuvant radiotherapy, found no difference in lymph node sampling $(P=.23)$ between obese $(\mathrm{BMI}>30)$ and nonobese. ${ }^{46}$ Another retrospective analysis that included 254 patients with rectal adenocarcinoma stratified lymph node yield by BMI, with obesity defined by BMI $>30 .{ }^{47}$ Two thirds of the patients were treated with neoadjuvant radiotherapy and/or chemotherapy, and a minority of patients underwent laparoscopic resection (4\% of obese, 
$11 \%$ nonobese). There was no difference in lymph node harvest in obese versus nonobese $(P=.57)$ patients, though in both groups the number of lymph nodes fell short of the recommended 12 nodes. There was no difference in tumor differentiation or staging according to $\mathrm{BMI}(P=.38)$.

The largest retrospective review included 596 patients operated after neoadjuvant radiotherapy and a variable regimen chemotherapy who underwent total mesorectal excision and were stratified based on BMI $\geq 30$ (obese) and BMI $<30$ (nonobese). The number of laparoscopic procedures was not reported. No significant difference in lymph node retrieval during surgery based on BMI was found, with an average number of lymph nodes retrieved $>12 .{ }^{48}$ Meyerhardt et al analyzed a nested cohort of 1,688 rectal cancer patients undergoing adjuvant chemo- and radiotherapy based on BMI. ${ }^{49}$ They, too, did not find any significant differences in lymph node retrieval across BMI ranges $(P=.75)$, with an average of nine lymph nodes retrieved in normal weight, obese, and overweight patients. There was also no difference in tumor differentiation.

Finally, a retrospective study from Hungary examined 141 patients who underwent surgery for rectal or rectosigmoid carcinoma without neoadjuvant therapy and did find a significant decrease in lymph node harvest in obese (BMI $>25$ ) versus nonobese patients ( 4.8 vs 7.5 nodes, $P=.004) .{ }^{50}$ However, this difference was observed only in what the authors defined as "short specimens" $(\leq 16 \mathrm{~cm})$. Seven percent of the specimens contained no lymph nodes at all. The average number of nodes detected in nonobese patients with long specimens $(>16 \mathrm{~cm})$ was also inadequate at 8.5 nodes. Thus, this study undersampled or underdetected lymph nodes, and cannot be used to draw conclusions.

Based on the literature available, it would appear that lymph node retrieval is not affected by obesity in either colon or rectal cancer. However, there are still unanswered questions regarding whether obesity affects the likelihood of lymph node positivity rates, the number of nodes involved, tumor grade, and overall tumor stage in various populations. More studies are needed, and studies that take into account measures of obesity other than, or in addition to BMI, might help in resolving these issues.

\section{Margins, Recurrence, and Survival}

A multitude of retrospective studies has examined the associations between obesity and margin positivity rates, recurrence, and survival after resection for colon and rectal cancers. Dignam et al examined a cohort of 4,288 patients who underwent resection of Duke B/C colon carcinoma and were randomized to participation in different National Surgical Adjuvant Breast and Bowel Project (NSABP) adjuvant chemotherapy trials. ${ }^{51}$ Aver- age follow-up was 11.2 years. They examined diseasefree survival, overall survival, second primary cancer, and colon cancer events across a range of BMIs. A hazard ratio of 1.27 (95\% CI, 1.05-1.53) for disease-free survival adverse event was incurred by those with $\mathrm{BMI}>35$; actual colon cancer event HR was 1.38 (95\% CI, 1.101.73). Overall mortality was also increased in this BMI group (HR, 1.28; 95\% CI, 1.04-1.57), increase in noncancer death risk was not significant, but the cancerrelated death risk was increased by $36 \%$ (HR, 1.36; 95\% CI, 1.06-1.73). ${ }^{51}$ Gender-based analysis did not yield any significant difference between men and women. Analysis by regression model controlled for the type of adjuvant chemotherapy. Of note, body surface area was capped at $2 \mathrm{~m}^{2}$ for the calculation of chemotherapy dosing, which may have resulted in under treatment of the very obese.

Regarding gender and obesity, in another study of 3,759 men and women undergoing various adjuvant chemotherapy regimens for resected stage II and III colon cancer, with a median follow-up of 9.4 years, combined analysis did not yield significance for either tumor recurrence or cancer survival, but when stratified for gender, women with $\mathrm{BMI} \geq 30$ had a higher risk for overall mortality (HR, 1.34,95\% CI, 1.07-1.67; $P=.007)$ and a nonsignificant trend for disease recurrence. $^{52}$ Obese men in this cohort did not have significantly different long-term outcomes compared with men with normal BMI.

A more recent retrospective analysis of 868 colon cancer patients randomized to different adjuvant 5-FUbased regimens found significant survival differences when patients were stratified for obesity, with a worse result in the obese cohort. ${ }^{41}$ Specifically, obese patients with a BMI $\geq 35$ were found to have worse outcome with regards to disease-free survival (HR, 1.23; 95\% CI, $1.01-1.49 ; P=.037)$ in univariate analysis. However, these patients varied considerably according to adjuvant regimen they received (1,155 received inadequate adjuvant therapy or no adjuvant treatment). A multivariate analysis revealed gender-specific differences: men with $\mathrm{BMI} \geq 35 \mathrm{had}$ worse overall survival (HR, $1.35 ; 95 \% \mathrm{CI}$, $1.02-1.79 ; P=.0391)$, and women with BMI between 30 and 34.9, but not with higher BMIs, had a worse risk for overall survival (HR, 1.24; 95\% CI, 1.01-1.53; $P=.0447) .{ }^{41}$ Another retrospective study of $1,053 \mathrm{pa}^{-}$ tients involved in stage III colon cancer adjuvant therapy trials analyzed the effects of increased BMI, and found no association between higher BMI and colon cancer recurrence or death. Gender stratification did not yield any difference in results ${ }^{43}$ in contrast to the data reported by Dignam et al as discussed above. ${ }^{51}$ In another study examining these issues, Healy et al examined the association of BMI with survival in a retrospective cohort of 414 patients with colorectal cancer with a median follow-up of 73 months. ${ }^{42}$ Notably, 337 patients had 
surgery alone, 77 received neoadjuvant therapy and surgery, while 179 received adjuvant chemotherapy and 35 had adjuvant radiotherapy. There was no difference in survival in obese versus nonobese for colon cancer, whereas for those patients in the rectal cancer group a statistical difference in survival $(P=.074 ; 71 \% 5$-year survival for nonobese and $59 \%$ survival for obese group) did approach significance. (There is no clear answer to this because the vast majority of results combine colon and rectal cancers' data. The authors state only that treatment of colon cancer was primarily by surgical resection with adjuvant chemotherapy for node-positive patients and for node negative patients with adverse pathologic features. Most rectal cancer patients with T3 or T4 tumors were offered neoadjuvant therapy involving a regimen of chemotherapy (5-fluorouracil [5-FL]) and radiation therapy (40-45 Gy in 20-25 fractions, but they don't say how many actually had it, or how many finished the full course).

Several studies have examined these issues in relation to rectal cancer alone. A recent retrospective review of 254 rectal cancer patients undergoing total mesorectal excision, two thirds of whom underwent neoadjuvant radio- or chemoradiotherapy and with a little more than half undergoing adjuvant chemo- or radiotherapy, there were no differences in complication rates or short-term mortality even when BMI was analyzed as a continuous variable. ${ }^{47}$ Obese patients in this study (BMI $\geq 30)$ had no worse 2-year overall survival, disease-free survival, local or distant recurrence. There was also no difference in margin negativity between obese and nonobese patients. Similar findings were offered by a retrospective review by Chern et al. ${ }^{48}$ These authors analyzed 596 patients undergoing total mesorectal excision after neoadjuvant chemoradiation. There was no difference in obese (BMI $\geq 30$ ) versus nonobese patients in the rate of positive or close margins, sphincter preservation, disease-free survival, or overall survival at either 39 months or 5 years, and local recurrence at 5 years. There were no differences between men and women. Meyerhardt et al, in contrast, found recurrence and sphincter preservation differences in obese men undergoing adjuvant chemoradiotherapy after rectal carcinoma operation in 1,688 patients. ${ }^{49}$ In particular, the OR of having an APR (abdominal perineal resection) in obese men (BMI $\geq 30)$ was 2.41 (95\% CI, 1.57-3.71). Local recurrence HR was 1.61 (95\% CI, 1.00-2.59) for obese men.

Finally, You et al published a retrospective analysis of 1,873 patients in whom the location of the rectal cancer was stratified into lower (within $10 \mathrm{~cm}$ of anal verge) and upper tumors (above $10 \mathrm{~cm}$ from anal verge). ${ }^{53}$ Only $9 \%$ patients had neoadjuvant therapy, and the median follow-up was 74.5 months. Obese patients did not have more lymph node metastasis or higher TNM stage, but obese patients did tend to more frequently have a narrow resection margin, defined as $<2 \mathrm{~cm}(67.7 \%$ in obese vs $77.2 \%$ in normal weight patients; $P=.019)$. There was no difference in diseasefree survival between obese and normal weight patients. However, stratification of tumors into upper and lower rectum yielded significant differences for lower rectum only: In multivariate analysis, obese male patients had higher rates of local recurrence compared with female patients (HR, 1.65; 95\% CI, 1.08-2.54; $P=.022$ ). Additionally, the risk of local recurrence for lower rectal tumors in this cohort increased in proportion to increasing BMI, reaching significance in obese patients (BMI $\geq 30 ; \mathrm{HR}, 4.71 ; 95 \% \mathrm{CI}, 1.01-21.09 ; P=.026)$. No such effects were recorded for upper rectal cancer cases.

Thus, there is no clear evidence that obesity predisposes to worse overall survival or increased recurrence in colorectal cancer. In studies involving patients who did not receive neoadjuvant treatment for rectal cancer, there may be a survival and local recurrence disadvantage in the obese, especially for lower rectum cancers. More studies are needed to address this question. The existing literature is composed of retrospective cohort reviews with varying chemotherapy regimens and a singular estimation of obesity by BMI calculation. The contradictory nature of the literature may be the result of treating $\mathrm{BMI}$ as a categorical variable where in fact it is a continuous variable, and a poor parameter for recognizing obesity in all-comers, ${ }^{54}$ as well as from insufficient data regarding cancer behavior in specific subsites, especially when it comes to comparing tumors in the upper versus the lower rectum. ${ }^{53}$

\section{Operative and Short-Term Effect of Obesity on Colorectal Cancer}

Most studies dedicated to these topics are retrospective cohort studies deriving their information from hospital registries or quality assurance project databases.

A 2009 retrospective case-control study of 3,202 patients in 121 hospitals undergoing colectomy for malignancy analyzed 30-day outcomes, and found that a BMI $\geq 35$ was significantly associated with all surgical infection, wound dehiscence (OR, 3.5), pulmonary embolism (OR, 6.98), urinary tract infection, and any complication, but not mortality. ${ }^{55}$

A retrospective review of 133 Japanese patients who underwent elective laparoscopic colectomy examined outcomes with obesity defined in two ways: (1) visceral obesity (visceral fat area (VFA) by CT scan calculation $\geq 130 \mathrm{~cm}^{2}$ ), and (2) general obesity (BMI $\left.\geq 25 \mathrm{~kg} / \mathrm{m}^{2}\right) .{ }^{54}$ There were more VFA obese than BMI obese. Median operative time was longer in VFA obese by 30 minutes $(P=.006)$ and in BMI obese by 27.5 minutes $(P=.01)$. Blood loss and conversion to laparotomy rates did not vary between obese and nonobese regardless of the definition of obesity. Wound infection 
rates and overall complications, as well as length of hospital stay were higher in VFA obese only, and did not come close to significance in BMI obese. Using the definitions of VFA and BMI obesity, the authors calculated that $44.3 \%$ of patients BMI-nonobese were VFA obese, and 22\% BMI-obese were VFA-nonobese. ${ }^{54}$ These results emphasize once again the critical shortcoming of most data on colorectal cancer and obesitythe very definition of obesity is not uniform across various geographic regions, populations, genders, and age groups.

Healy et al, in contrast, did not find any significant association between postoperative complications, 30 -day mortality, and obesity. ${ }^{42}$ In a retrospective review of 401 patients undergoing colectomy (68\% open approach, 26\% laparoscopic approach), operative time steadily increased with increasing BMI, and was one hour longer comparing morbidly obese to underweight patients. ${ }^{44}$ A systematic review by a New Zealand group addressed the issue of operative time in colon cases and found that increases as likely prolonged for male pelvis and complicated procedures versus female pelvis and short procedures. ${ }^{56}$

Short-term morbidity for rectal cancer resection seems to be increased with obesity. In addition, obesity is reported to be significantly associated with rates of anastomotic leak, ${ }^{57,58}$ parastomal hernia, ${ }^{59}$ wound infection, ${ }^{60,61}$ length of laparoscopic surgery according to $\mathrm{BMI},{ }^{46-48,60}$ length of laparoscopic surgery according to visceral adipose area, ${ }^{62,63}$ overall and systemic complications, ${ }^{62}$ more frequent conversion to laparotomy, ${ }^{46,64}$ more blood loss, ${ }^{47,58,63}$ and longer hospital stay. ${ }^{48}$ In addition, obese patients undergoing laparoscopic colorectal surgery are at an increased risk for conversion to open operation and operative times are significantly increased for obese patients having rectal cancer surgery. ${ }^{56}$ Having said this, however, the literature is not definitive as far as overall mortality and morbidity related to colon cancer surgery in the obese, though the obese do seem to incur proportionally more morbidity and mortality as a result of rectal cancer surgery.

\section{OBESITY AND CHEMOTHERAPY AND RADIATION THERAPY}

\section{Nonalcoholic Fatty Liver Disease}

Obesity and nonalcoholic fatty liver disease (NAFLD) are linked, with both being components of the metabolic syndrome. It is estimated that $30 \%$ of the United States general population has excessive fat accumulation in the liver (steatosis), and the number may be as high as 75 to $100 \%$ among the obese and morbidly obese. ${ }^{65}$ One study estimated the prevalence of NAFLD as a function of BMI: BMI $\leq 25$ translated to a prevalence of $24.5 \%, 25$ $<$ BMI $<30$ had a $67 \%$ rate of NAFLD, and in those with $\mathrm{BMI}>30$, the rate of NAFLD was $91 \%{ }^{66}$ The molecular characteristic of NAFLD is an increased accumulation of triglycerides in hepatocytes. Hepatic triglyceride accumulation can lead to mitochondrial dysfunction; although most patients with NAFLD are asymptomatic, $\sim 2$ to $6 \%$ of the general U.S. population and up to $20 \%$ of the obese may progress to steatosis with inflammation, fibrosis, and cirrhosis. ${ }^{65}$ The low-grade inflammatory state and insulin resistance associated with obesity may contribute to this progression to nonalcoholic steatohepatitis (NASH). Liver biopsy is considered the gold standard for diagnosis, though it is invasive and impractical. There is no approved treatment for NAFLD or NASH.

\section{Chemotherapy-Associated Hepatotoxicity in the Obese}

In patients with colorectal liver metastases, treatment with chemotherapy is associated with liver injury in up to 20 to $30 \%$ of patients. ${ }^{67}$ Irinotecan in particular is associated with steatosis and steatohepatitis, whereas oxaliplatin is associated with grade 3 sinusoidal dilation. ${ }^{67} 5-\mathrm{FU}$, the base for chemotherapy regimens for colorectal cancer is also associated with steatosis. ${ }^{68} \mathrm{It}$ is unknown if hepatic steatosis is induced by chemotherapy or exacerbated by it. ${ }^{68}$ Taking into account the prevalence of obesity in the developed world along with the high incidence of colorectal cancer, steatosis, and NAFLD, patients with colorectal metastases who are obese, diabetic, or carry a diagnosis of metabolic syndrome, should be given special consideration in view of the increased incidence of steatosis and steatohepatitis associated with irinotecan.

A study by Vauthey et al examined a cohort of 406 patients undergoing hepatic resection for colorectal metastases. ${ }^{69}$ Most of them received preoperative chemotherapy. Irinotecan therapy was significantly associated with steatohepatitis compared with no neoadjuvant therapy (OR, 5.4; 95\% CI, 2.2-13.5). Moreover, patients with steatohepatitis had an increased 90-day mortality compared with patients without the condition (14.7\% vs $1.6 \%$; OR, 10.5 ; 95\% CI, 2.0-36.4). When BMI was taken into account, the authors concluded that irinotecan had a strong association with steatohepatitis regardless of BMI, but the effect was more pronounced in patients with a higher BMI (BMI $<2512.1 \%$ steatohepatitis vs $\mathrm{BMI} \geq 25, \quad 24.6 \%$ steatohepatitis, $P=.01)^{69}$

A smaller study also found a significant association with irinotecan and steatohepatits. ${ }^{70}$ In this study, multivariate analysis revealed that irinotecan and BMI were both independent predictors of NASH.

Steatosis associated with obesity, irinotecan, and 5-FU incurred additional risk of morbidity, mainly from infection, but not mortality. ${ }^{66}$ Steatohepatitis was 
associated with irinotecan therapy and was shown to be higher in patients with $\mathrm{BMI} \geq 25$, with increased mortality. These factors should be kept in mind and may limit consideration for neoadjuvant chemotherapy in colorectal cancer metastasis in patients with $\mathrm{BMI} \geq 25$ and those with known liver steatosis.

\section{Diabetes and Response to Chemotherapy and/ or Radiotherapy}

Because there is such a high prevalence of diabetes in the obese population, we must briefly discuss its interaction with chemotherapy and radiotherapy. Caudle et al addressed this in a retrospective review of 110 patients with rectal cancer undergoing neoadjuvant chemoradiotherapy. ${ }^{71}$ Of the 110 patients in this cohort, 17 were diabetic. Although tumor downstaging rates were similar, nodal downstaging was nonsignificantly better in nondiabetics. None of the diabetic patients achieved a complete response, whereas $23 \%$ of nondiabetics did $(P=.039)$. In addition, diabetics had higher rates of local progression ( $24 \%$ vs $5 \% ; P=.046)$. Unfortunately, this study was underpowered due to the small number of diabetic patients. This study is an indication that there may indeed be a link between nodal resistance to chemoradiotherapy and diabetes in rectal cancer patients. There is not enough data to say for sure what the differences are. One of the main issues is that the response of adipose tissue to radiation has not been well studied; meanwhile, adipose stores serve as an extra endocrine organ in the body, and what happens to various hormonal and cytokine productions after it has been irradiated, is not known. In another study examining the use of neoadjuvant chemoradiotherapy in the treatment of rectal cancer, the authors found that in 157 rectal cancer patients treated with an abdominoperineal resection, with $45 \%$ of patients treated with neoadjuvant radiotherapy with or without chemotherapy, ${ }^{72}$ wound infection rates were statistically significantly worse in patients with BMI $>30 \mathrm{~kg} / \mathrm{m}^{2}$. These associations need to be explored in further studies.

The issue of colon cancer outcome and obesity was examined by Meyerhardt et al. In a cohort of 3,549 patients with stage II and III colon cancer treated with adjuvant chemotherapy, 287 patients were diabetic. With over 9.4 years of follow-up, these authors reported that nondiabetic patients were significantly more likely to survive without recurrence than diabetic subjects. ${ }^{73} \mathrm{In}$ addition, compared with nondiabetics, diabetics had a $42 \%$ higher risk of death from any cause $(P<.0001)$.

To summarize, there is no doubt that colorectal cancer is an obesity-associated disease. The mechanisms are many, including insulin and insulin-like growth factor 1 and its receptors, adipocytokines and sex hormone from fat tissue, etc. In population studies, obese men carry a higher risk of colorectal cancer, whereas obese women carry a higher risk of colon cancer only. The benefit of exercise cannot be underestimated, especially in the obese, as it has the potential to significantly reduce the risk of $\mathrm{CRC}$ without necessarily having an impact on BMI. BMI itself is not a reliable measure in defining obesity, and visceral fat accumulation and anthropometric waist circumference and waist-to-hip ratio are perhaps better suited, though they have flaws of their own. In truth, there is no one measure of obesity that can be applied to all populations, genders, and age groups. In addition, diabetes is emerging as a novel major risk factor for colorectal cancer. There is a major need for well-designed American studies of diabetic patients with emphasis on indices of glycemic control, type of therapy, onset of disease, and multiple definitions of weight categories to determine whether earlier screening or shorter intervals between screenings would be cost effective.

\section{REFERENCES}

1. Sikalidis AK, Varamini B. Roles of hormones and signaling molecules in describing the relationship between obesity and colon cancer. Pathol Oncol Res 2011;17(4):785-790

2. Giovannucci E. Nutrition, insulin, insulin-like growth factors and cancer. Horm Metab Res 2003;35(11-12):694-704

3. Warren RS, Yuan H, Matli MR, Ferrara N, Donner DB. Induction of vascular endothelial growth factor by insulinlike growth factor 1 in colorectal carcinoma. J Biol Chem 1996;271(46):29483-29488

4. Rokkas T, Pistiolas D, Sechopoulos P, Margantinis G, Koukoulis G. Risk of colorectal neoplasm in patients with acromegaly: a meta-analysis. World J Gastroenterol 2008; 14(22):3484-3489

5. Jaffe T, Schwartz B. Leptin promotes motility and invasiveness in human colon cancer cells by activating multiple signal-transduction pathways. Int J Cancer 2008;123(11): 2543-2556

6. Rasouli N, Kern PA. Adipocytokines and the metabolic complications of obesity. J Clin Endocrinol Metab 2008; 93(11, Suppl 1):S64-S73

7. Martínez ME, Heddens D, Earnest DL, et al. Physical activity, body mass index, and prostaglandin E2 levels in rectal mucosa. J Natl Cancer Inst 1999;91(11):950-953

8. Takahashi H, Takayama T, Yoneda K, et al. Association of visceral fat accumulation and plasma adiponectin with rectal dysplastic aberrant crypt foci in a clinical population. Cancer Sci 2009;100(1):29-32

9. Amemori S, Ootani A, Aoki S, et al. Adipocytes and preadipocytes promote the proliferation of colon cancer cells in vitro. Am J Physiol Gastrointest Liver Physiol 2007; 292(3):G923-G929

10. Slattery ML, Ballard-Barbash R, Edwards S, Caan BJ, Potter JD. Body mass index and colon cancer: an evaluation of the modifying effects of estrogen (United States). Cancer Causes Control 2003;14(1):75-84

11. Nguyen NT, Nguyen XMT, Lane J, Wang P. Relationship between obesity and diabetes in a US adult population: findings from the National Health and Nutrition Examination Survey, 1999-2006. Obes Surg 2011;21(3):351-355 
12. Siddiqui AA, Spechler SJ, Huerta S, Dredar S, Little BB, Cryer B. Elevated HbA1c is an independent predictor of aggressive clinical behavior in patients with colorectal cancer: a case-control study. Dig Dis Sci 2008;53(9):2486-2494

13. Giouleme O, Diamantidis MD, Katsaros MG. Is diabetes a causal agent for colorectal cancer? Pathophysiological and molecular mechanisms World J Gastroenterol 2011;17(4): 444-448

14. Larsson SC, Rutegård J, Bergkvist L, Wolk A. Physical activity, obesity, and risk of colon and rectal cancer in a cohort of Swedish men. Eur J Cancer 2006;42(15):25902597

15. Chao A, Connell CJ, Jacobs EJ, et al. Amount, type, and timing of recreational physical activity in relation to colon and rectal cancer in older adults: the Cancer Prevention Study II Nutrition Cohort. Cancer Epidemiol Biomarkers Prev 2004;13(12):2187-2195

16. Isomura K, Kono S, Moore MA, et al. Physical activity and colorectal cancer: the Fukuoka Colorectal Cancer Study. Cancer Sci 2006;97(10):1099-1104

17. Harriss DJ, Atkinson G, George K, et al; C-CLEAR group. Lifestyle factors and colorectal cancer risk (1): systematic review and meta-analysis of associations with body mass index. Colorectal Dis 2009;11(6):547-563

18. Deurenberg P, Yap M, van Staveren WA. Body mass index and percent body fat: a meta-analysis among different ethnic groups. Int J Obes Relat Metab Disord 1998;22(12):11641171

19. Deurenberg P, Deurenberg-Yap M, Guricci S. Asians are different from Caucasians and from each other in their body mass index/body fat percent relationship. Obes Rev 2002; 3(3):141-146

20. Razak F, Anand SS, Shannon H, et al. Defining obesity cut points in a multiethnic population. Circulation 2007;115(16): 2111-2118

21. Low S, Chin MC, Ma S, Heng D, Deurenberg-Yap M. Rationale for redefining obesity in Asians. Ann Acad Med Singapore 2009;38(1):66-69

22. World Health Organization. Available at: http://www.who. int/mediacentre/factsheets/fs311/en/index.html. Accessed March 9, 2011

23. Lear SA, James PT, Ko GT, Kumanyika S. Appropriateness of waist circumference and waist-to-hip ratio cutoffs for different ethnic groups. Eur J Clin Nutr 2010;64(1):42-61

24. Yoshizumi T, Nakamura T, Yamane M, et al. Abdominal fat: standardized technique for measurement at CT. Radiology 1999;211(1):283-286

25. Yoon DY, Moon JH, Kim HK, et al. Comparison of lowdose CT and MR for measurement of intra-abdominal adipose tissue: a phantom and human study. Acad Radiol 2008;15(1):62-70

26. Carroll JF, Chiapa AL, Rodriquez M, et al. Visceral fat, waist circumference, and BMI: impact of race/ethnicity. Obesity (Silver Spring) 2008;16(3):600-607

27. Bassett JK, Severi G, English DR, et al. Body size, weight change, and risk of colon cancer. Cancer Epidemiol Biomarkers Prev 2010;19(11):2978-2986

28. Thygesen LC, Grønbaek M, Johansen C, Fuchs CS, Willett WC, Giovannucci E. Prospective weight change and colon cancer risk in male US health professionals. Int J Cancer 2008;123(5):1160-1165

29. Pischon T, Lahmann PH, Boeing $\mathrm{H}$, et al. Body size and risk of colon and rectal cancer in the European Prospective
Investigation into Cancer and Nutrition (EPIC). J Natl Cancer Inst 2006;98(13):920-931

30. Larsson SC, Wolk A. Obesity and colon and rectal cancer risk: a meta-analysis of prospective studies. Am J Clin Nutr 2007;86(3):556-565

31. Dai Z, Xu YC, Niu L. Obesity and colorectal cancer risk: a meta-analysis of cohort studies. World J Gastroenterol 2007;13(31):4199-4206

32. Moghaddam AA, Woodward M, Huxley R. Obesity and risk of colorectal cancer: a meta-analysis of 31 studies with 70,000 events. Cancer Epidemiol Biomarkers Prev 2007;16(12): 2533-2547

33. Oxentenko AS, Bardia A, Vierkant RA, et al. Body size and incident colorectal cancer: a prospective study of older women. Cancer Prev Res (Phila) 2010;3(12):1608-1620

34. Geer EB, Shen W. Gender differences in insulin resistance, body composition, and energy balance. Gend Med 2009;6(1, Suppl 1):60-75

35. Berster JM, Göke B. Type 2 diabetes mellitus as risk factor for colorectal cancer. Arch Physiol Biochem 2008;114(1):8498

36. Larsson SC, Orsini N, Wolk A. Diabetes mellitus and risk of colorectal cancer: a meta-analysis. J Natl Cancer Inst 2005; 97(22):1679-1687

37. Oh SW, Kim YH, Choi YS, et al. The comparison of the risk factors and clinical manifestations of proximal and distal colorectal cancer. Dis Colon Rectum 2008;51(1):5661

38. Yang YX, Hennessy S, Lewis JD. Insulin therapy and colorectal cancer risk among type 2 diabetes mellitus patients. Gastroenterology 2004;127(4):1044-1050

39. Rex DK, Johnson DA, Anderson JC, Schoenfeld PS, Burke CA, Inadomi JM; American College of Gastroenterology. American College of Gastroenterology guidelines for colorectal cancer screening 2009 [corrected]. Am J Gastroenterol 2009;104(3):739-750

40. Serrano MJ, Carrasco MA, Ortiz R, Cazador AC. [The impact of obesity on the histopathological characteristics of colorectal tumours. An observational study]. Cir Esp 2010; 87(1):33-38

41. Sinicrope FA, Foster NR, Sargent DJ, O'Connell MJ, Rankin C. Obesity is an independent prognostic variable in colon cancer survivors. Clin Cancer Res 2010;16(6):18841893

42. Healy LA, Ryan AM, Sutton E, et al. Impact of obesity on surgical and oncological outcomes in the management of colorectal cancer. Int J Colorectal Dis 2010;25(11):12931299

43. Meyerhardt JA, Niedzwiecki D, Hollis D, et al; Cancer and Leukemia Group B 89803. Impact of body mass index and weight change after treatment on cancer recurrence and survival in patients with stage III colon cancer: findings from Cancer and Leukemia Group B 89803. J Clin Oncol 2008; 26(25):4109-4115

44. Linebarger JH, Mathiason MA, Kallies KJ, Shapiro SB. Does obesity impact lymph node retrieval in colon cancer surgery? Am J Surg 2010;200(4):478-482

45. Damadi AA, Julien L, Arrangoiz R, Raiji M, Weise D, Saxe AW. Does obesity influence lymph node harvest among patients undergoing colectomy for colon cancer? Am Surg 2008;74(11):1073-1077

46. Bège T, Lelong B, Francon D, Turrini O, Guiramand J, Delpero JR. Impact of obesity on short-term results of 
laparoscopic rectal cancer resection. Surg Endosc 2009; 23(7): 1460-1464

47. Ballian N, Yamane B, Leverson G, et al. Body mass index does not affect postoperative morbidity and oncologic outcomes of total mesorectal excision for rectal adenocarcinoma. Ann Surg Oncol 2010;17(6):1606-1613

48. Chern H, Chou J, Donkor C, et al. Effects of obesity in rectal cancer surgery. J Am Coll Surg 2010;211(1):55-60

49. Meyerhardt JA, Tepper JE, Niedzwiecki D, et al. Impact of body mass index on outcomes and treatment-related toxicity in patients with stage II and III rectal cancer: findings from Intergroup Trial 0114. J Clin Oncol 2004;22(4):648-657

50. Görög D, Nagy P, Péter A, Perner F. Influence of obesity on lymph node recovery from rectal resection specimens. Pathol Oncol Res 2003;9(3):180-183

51. Dignam JJ, Polite BN, Yothers G, et al. Body mass index and outcomes in patients who receive adjuvant chemotherapy for colon cancer. J Natl Cancer Inst 2006;98(22):1647-1654

52. Meyerhardt JA, Catalano PJ, Haller DG, et al. Influence of body mass index on outcomes and treatment-related toxicity in patients with colon carcinoma. Cancer 2003;98(3):484495

53. You JF, Tang R, Changchien CR, et al. Effect of body mass index on the outcome of patients with rectal cancer receiving curative anterior resection: disparity between the upper and lower rectum. Ann Surg 2009;249(5):783-787

54. Tsujinaka S, Konishi F, Kawamura YJ, et al. Visceral obesity predicts surgical outcomes after laparoscopic colectomy for sigmoid colon cancer. Dis Colon Rectum 2008;51(12):17571765; discussion 1765-1767

55. Merkow RP, Bilimoria KY, McCarter MD, Bentrem DJ. Effect of body mass index on short-term outcomes after colectomy for cancer. J Am Coll Surg 2009;208(1):53-61

56. Gendall KA, Raniga S, Kennedy R, Frizelle FA. The impact of obesity on outcome after major colorectal surgery. Dis Colon Rectum 2007;50(12):2223-2237

57. Asteria CR, Gagliardi G, Pucciarelli S, et al. Anastomotic leaks after anterior resection for mid and low rectal cancer: survey of the Italian Society of Colorectal Surgery. Tech Coloproctol 2008;12(2):103-110

58. Benoist S, Panis Y, Alves A, Valleur P. Impact of obesity on surgical outcomes after colorectal resection. Am J Surg 2000;179(4):275-281

59. De Raet J, Delvaux G, Haentjens P, Van Nieuwenhove Y. Waist circumference is an independent risk factor for the development of parastomal hernia after permanent colostomy. Dis Colon Rectum 2008;51(12):1806-1809

60. Balentine CJ, Wilks J, Robinson C, et al. Obesity increases wound complications in rectal cancer surgery. J Surg Res 2010;163(1):35-39
61. El-Gazzaz G, Kiran RP, Lavery I. Wound complications in rectal cancer patients undergoing primary closure of the perineal wound after abdominoperineal resection. Dis Colon Rectum 2009;52(12):1962-1966

62. Ishii Y, Hasegawa H, Nishibori H, Watanabe M, Kitajima M. Impact of visceral obesity on surgical outcome after laparoscopic surgery for rectal cancer. Br J Surg 2005;92(10): 1261-1262

63. Sakai T, Maekawa T, Mikami K, Kuramochi H, Noda S. Visceral fat volume and surgical outcomes of colorectal resection. Int Surg 2009;94(4):370-372

64. Singh A, Girivasan M, Pawa N, Riaz AA, Hendricks JB, Motson RW. Laparoscopic colorectal cancer surgery in obese patients. Colorectal Dis 2011;13(8):878-883

65. Rector RS, Thyfault JP, Wei Y, Ibdah JA. Non-alcoholic fatty liver disease and the metabolic syndrome: an update. World J Gastroenterol 2008;14(2):185-192

66. Khan AZ, Morris-Stiff G, Makuuchi M. Patterns of chemotherapy-induced hepatic injury and their implications for patients undergoing liver resection for colorectal liver metastases. J Hepatobiliary Pancreat Surg 2009;16(2):137144

67. Pawlik TM, Olino K, Gleisner AL, Torbenson M, Schulick R, Choti MA. Preoperative chemotherapy for colorectal liver metastases: impact on hepatic histology and postoperative outcome. J Gastrointest Surg 2007;11(7):860-868

68. Aloia TA, Fahy BN. Chemotherapy-associated hepatotoxicity: how concerned should we be? Expert Rev Anticancer Ther 2010;10(4):521-527

69. Vauthey JN, Pawlik TM, Ribero D, et al. Chemotherapy regimen predicts steatohepatitis and an increase in 90-day mortality after surgery for hepatic colorectal metastases. J Clin Oncol 2006;24(13):2065-2072

70. Fernandez FG, Ritter J, Goodwin JW, Linehan DC, Hawkins WG, Strasberg SM. Effect of steatohepatitis associated with irinotecan or oxaliplatin pretreatment on resectability of hepatic colorectal metastases. J Am Coll Surg 2005;200(6):845-853

71. Caudle AS, Kim HJ, Tepper JE, et al. Diabetes mellitus affects response to neoadjuvant chemoradiotherapy in the management of rectal cancer. Ann Surg Oncol 2008; 15(7):1931-1936

72. Zorcolo L, Restivo A, Capra F, Fantola G, Marongiu L, Casula G. Does long course radiotherapy influence postoperative perineal morbidity after abdominoperineal resection of the rectum for cancer? Colorectal Dis 2010 [Epub ahead of print]

73. Meyerhardt JA, Catalano PJ, Haller DG, et al. Impact of diabetes mellitus on outcomes in patients with colon cancer. J Clin Oncol 2003;21(3):433-440 